\title{
Capturing Membrane Trafficking Events During 3D Angiogenic Development in Vitro
}

Caitlin R. Francis ${ }^{1}$ and Erich J. Kushner ${ }^{1 *}$

${ }^{1}$ Department of Biological Sciences, University of Denver, Denver, CO

*Author for correspondence:

Erich J. Kushner

University of Denver

Department of Biological Sciences

Denver, CO 80210

Phone: 303-871-4386

Email: Erich.Kushner@du.edu

Abstract: 334 words

Manuscript: 4,955 words (not including figure legends or references)

Figures: 8

Supplemental Materials: 1 document, 6 movies

Running Title: Imaging Trafficking Events During Angiogenic Development In Vitro

Keywords: Imaging, sprouting, Trafficking, Exocytosis, Vesicle, Angiogenesis, Blood vessel, Vascular, Endothelial, Endothelium, Lumen, Development, Rab27a, apical membrane, von Willebrand factor, Weibel-Palade Body
Abbreviations:
2-dimensional (2D)
3-dimensional (3D)
Weibel Palade Body (WPB)
Von Willebrand Factor (VWF) 


\section{ABSTRACT}

\section{Objectives}

42 Mechanisms that govern angiogenesis are paramount to our understanding of how blood vessels

43 are formed embryonically, maintained in adulthood and manifest disease. Akin to transcriptional

44 regulation of endothelial-specific genes, vesicular trafficking events dictate protein localization,

45 functional activity, and half-life, providing a critically important regulatory step. However, there is

46 little information detailing endothelial-specific trafficking signatures. This is due, in part, by

47 limitations in visualizing trafficking events in endothelial tissues. Our aim in this investigation was

48 to explore the use of a 3-dimensional (3D) in vitro sprouting model to image and evaluate

49 membrane trafficking events compared to the conventional 2-dimensional (2D)-based culture

50 method.

Methods

53 Primary Human endothelial cells were challenged to make multicellular sprouts using a fibrin-

54 bead assay. An assortment of cell polarity and Rab proteins were quantified via

55 immunocytochemistry and live-imaging to compare their localization between 3D sprouts and 2D

56 culture.

\section{Results}

59 Our results show that sprouts generated from the fibrin-bead assay grow close to the imaging 60 plane allowing for an orthogonal view of apical and basal membrane domains. Compared with 2D 61 culture in which the apical and basal domains are in the axial orientation, limiting resolution, 3D

62 sprouts are acquired in the $X-Y$ plane providing high-resolution for viewing trafficking events.

63 Second, we demonstrate that fibrin-bead generated sprouts have a strong apicobasal polarity

64 axis. Third, we directly compare imaging of trafficking mediators podocalyxin and Rab35 between

$653 D$ sprouts and 2D culture. Here, we show that 3D sprouting structures are well-suited to capture 
66 trafficking events that are not present in 2D culture due to the lack of a defined apical domain.

67 Lastly, we compared exocytic events of von Willebrand Factor between 3D sprouting and 2D

68 culture. Our results demonstrate a distinct imaging advantage for monitoring these trafficking

69 programs in 3D sprouts as compared with conventional 2D culture.

70

71 Conclusions

72 In general, our results establish that the fibrin-bead sprouting assay is well-suited for sub-cellular

73 imaging of trafficking events during angiogenic growth. Additionally, the 2D endothelial culture

74 does not enforce the formation of an apicobasal polarity axis. 


\section{INTRODUCTION}

The process of early blood vessel formation, or angiogenesis, is critical to establish the

78 requisite vasculature for organismal growth [1]. During angiogenesis, early blood vessels

79 establish the canonical morphologies that define adult vasculature, namely long interconnected

80 conduits providing a portal for blood flow [2]. Endothelial cells are the initial building blocks of

81 blood vessel development, forming small sprouts and capillary-like networks that progressively

82 invade growing embryonic tissue. Our more recent ability to image gross angiogenic processes

83 in both development and disease has unlocked many outstanding questions in the field. This has

84 also been aided by the expanding wealth of transgenic animals as well as a multitude of cellular

85 and molecular biology techniques. However, many processes that are uniquely governed by

86 endothelial tissues and fundamental to their function remain to be explored. In particular,

87 membrane trafficking-based regulation of endothelial function is an underserved area of

exploration in the field of angiogenesis that is largely dependent on sub-cellular imaging techniques to interrogate function.

Trafficking broadly refers to the vesicle-based transport and movement of proteins through

91 the cell. This process is parsed into endocytic (outside material in), exocytic (inside material out),

92 and recycling (moving between both endocytic and exocytic) pathways [3-5]. Rab GTPase

93 proteins are the most recognized multifunctional mediators of all trafficking events acting as an

94 intracellular barcoding system with over 70 known family members $[6,7]$. Rab proteins attach to

95 the outside of vesicles where they define intracellular trafficking routes [8, 9]. Activated Rabs then

96 recruit or bind to tissue-specific effectors which can then promote localization, signaling, and

97 degradation of their cargo. For example, in ECs Rab27a is required for Weibel-Palade body

98 (WPB) apical membrane fusion and von Willebrand Factor (vWF) exocytosis [10]. Indeed, the

99 breadth of function trafficking encompasses is far and wide, and may collectively represent the

100 most dominant, yet esoteric, regulatory program in a protein's life cycle [7, 11, 12]. Taken as a 
101 whole, how endothelial tissues harness trafficking-based regulation is a major outstanding 102 question in the field of blood vessel development and homeostasis.

103 Much of the seminal work in the field of membrane trafficking has been carried out in 104 epithelial tissue [13]. This is in large part due to their large rectangular shape and spatially 105 segregated apical and basal domains allowing for relatively easy imaging of processes at either 106 membrane. Additionally, epithelial cells readily set up apicobasal polarity in 2D culture, thus do 107 not require much in the way of physical or chemical cues to elicit a defined polarity axis [14]. By 108 contrast endothelial cells are exceedingly flat exhibiting a mesenchymal morphology [15]. In some 109 instances, the distance between the apical and basal domains in endothelial cells are diffraction 110 limited $(\leq 500 \mathrm{~nm})$ hindering imaging of either membrane surface. In 2D culture endothelial cells 111 are highly migratory, setting up a defined planar cell polarity axis; however, removed from a 112 sprouting structure endothelial cells on a dish do not show a commitment to an apical and basal 113 membrane identity. Because trafficking biology primarily entails movement of vesicular cargo, 114 transcript levels of trafficking-mediators are typically held at a constant, excluding the use of 115 simple expression analysis to interrogate function. In this realm, the ability to visualize differential 116 trafficking events to various membranes is paramount to understanding function. Unfortunately, 117 in vivo imaging of endothelial trafficking events present a significant challenge, due to the relative 118 thinness of endothelial cells coupled with the requirement for a less magnified microscope 119 objective (e.g. $20 x$ vs $60 x$ lens) to span the tissue depth needed to capture blood vessels. 120 Therefore, we believe the use of a $3 D$ sprouting model is an excellent compromise between 121 providing the necessary cellular cues to reproduce angiogenic morphodynamics with ample sub122 cellular imaging accessibility to image trafficking events.

123 In this investigation our aim was to explore the use of a 3-dimensional (3D) in vitro 124 sprouting model to image and evaluate membrane trafficking events in two scenarios. First, our 125 goal was to capture sub-cellular dynamics of apical proteins, in particular Rab GTPases and their 126 cargo, related to lumen biogenesis. Second, was to evaluate luminal exocytic events at the apical 
127 membrane. Our reasoning for choosing these trafficking events was two-fold: 1) these events are

128 hard-to-impossible to distinguish in most in vivo models; and 2) are typically evaluated using 2-

129 dimensional (2D) culture. Using a fibrin-bead sprouting assay we demonstrate the utility of this

$1303 \mathrm{D}$ in vitro system for capturing endothelial-specific trafficking events. Methodologically, we

131 demonstrate that endothelial sprouts develop parallel and close to the imaging window allowing

132 for use of commonly equipped high-resolution objectives. Additionally, we show that lumen

133 biogenesis and exocytic trafficking events are easily imaged in multicellular sprouts to a much

134 greater extent than 2D culture. Overall, this work highlights a highly reproducible in vitro assay

135 that provides a tailored imaging platform for exploring blood vessel-specific trafficking networks.

\section{MATERIALS AND METHODS}

A list of used materials is included in the Supplemental Data. The authors will make their raw data, analytic methods, and study materials available to other researchers upon written 140 request.

\section{Cell culture.}

Pooled Human umbilical vein endothelial cells (HUVECs) were purchased from PromoCell

144 and cultured in EGM-2 media (PromoCell Growth Medium, ready-to-use) for 2-5 passages. For

145 experiments glass-bottomed imaging dishes were exposed to deep UV light for 6 minutes and 146 coated with Poly-D-Lysine (ThermoFisher) for a minimum of 20 minutes. Small interfering RNA 147 (ThermoFisher) was introduced into primary HUVEC using the Neon® transfection system 148 (ThermoFisher). Scramble and Rab27a siRNAs were purchased from (ThermoFisher) and 149 resuspended to a $10 \mu \mathrm{M}$ stock concentration and used at $0.5 \mu \mathrm{M}$ (see data supplement). Normal 150 human lung fibroblasts (NHLFs, Lonza) and HEK-A (ThermoFisher) were maintained in 151 Dulbeccos Modified Medium (DMEM) supplemented with 10\% fetal bovine serum and pen/strep 
152 antibiotics. Both NHLFs and HEKs were used up to 15 passages. All cells were maintained in a

153 humidified incubator at $37^{\circ} \mathrm{C}$ and $5 \% \mathrm{CO}_{2}$.

\section{Sprouting Angiogenesis Assay.}

Fibrin-bead assay was performed as originally reported by Nakatsu et al. 2007 [16].

157 Briefly, HUVECs were coated onto microcarrier beads (Amersham) and plated overnight. SiRNA-

158 treatment or viral transduction was performed the same day the beads were coated. The following

159 day, the EC-covered microbeads were embedded in a fibrin matrix. Once the clot was formed,

160 media was overlaid along with 100,000 NHLFs. Media was changed daily along with monitoring

161 of sprout development.

Plasmid Constructs.

The following constructs were procured for the study: GFP-Rab27A (gift from William

165 Gahl; Addgene plasmid \#89237); Rab35 (gift from Peter McPherson; Addgene plasmid \#47424);

166 Neo DEST (705-1) (gift from Eric Campeau \& Paul Kaufman; Addgene plasmid \#17392);

167 pMDLg/pRRE (gift from Didier Trono; Addgene plasmid \# 12251); pVSVG (gift from Bob

168 Weinberg; Addgene plasmid \#8454 ); psPAX2 (gift from Didier Trono; Addgene plasmid \#12260);

169 pShuttle-CMV (gift from Bert Vogelstein; Addgene plasmid \#16403); and AdEasier-1 cells (gift

170 from Bert Vogelstein; Addgene, \#16399)

172 Lentivirus and Adenovirus Generation.

173 Lentivirus was generated by using the LR Gateway Cloning method [17]. Genes of interest

174 and fluorescent proteins were isolated and incorporated into a pME backbone via Gibson reaction

175 [18]. Following confirmation of the plasmid by sequencing the pME entry plasmid was mixed with

176 the destination vector and LR Clonase. The destination vector used in this study was pLenti CMV

177 Neo DEST (705-1). Once validated, the destination plasmids were transfected with the three 
178 required viral protein plasmids: pMDLg/pRRE, pVSVG and psPAX2 into HEK 293 cells. The

179 transfected HEKs had media changed 4 hours post transfection and the viral media was 180 harvested at day 3.

181 Adenoviral constructs and viral particles were created using the Adeasy viral cloning 182 protocol [19]. Briefly, transgenes were cloned into a pShuttle-CMV plasmid via Gibson Assembly.

183 PShuttle-CMV plasmids were then digested overnight with Mssl (ThermoFisher) and linearized

184 pShuttle-CMV plasmids were transformed into the final viral backbone using electrocompetent

185 AdEasier-1 cells. Successful incorporation of pShuttle-CMV construct into AdEasier-1 cells were 186 confirmed via digestion with Pacl (ThermoFisher). $5000 \mathrm{ng}$ plasmid was then digested at $37^{\circ} \mathrm{C}$ 187 overnight, then $85^{\circ} \mathrm{C}$ for 10 minutes and transfected in a $3: 1$ polyethylenimine (PEI, Sigma):DNA ratio into $70 \%$ confluent HEK $293 \mathrm{~A}$ cells (ThermoFisher) in a T-25 flask.

Over the course of 2-4 weeks, fluorescent cells became swollen and budded off the plate. at $2000 \mathrm{rpm}$ for 5 minutes in a $15 \mathrm{~mL}$ conical tube. The supernatant was aspirated, and cells were

192 resuspended in $1 \mathrm{~mL}$ PBS. Cells were then lysed by 3 consecutive quick freeze-thaw cycles in 193 liquid nitrogen, spun down for 5 minutes at $2000 \mathrm{rpm}$, and supernatant was added to $70 \%$ 194 confluent T-75 flasks. Propagation continued and collection repeated for infection of $10-15 \mathrm{~cm}$ 195 dishes. After collection and 4 freeze thaw cycles of virus collected from $10-15 \mathrm{~cm}$ dishes, $8 \mathrm{~mL}$ 196 viral supernatant was collected and combined with $4.4 \mathrm{~g} \mathrm{CsCl}$ (Sigma) in $10 \mathrm{~mL}$ PBS. Solution 197 was overlaid with mineral oil and spun at $32,000 \mathrm{rpm}$ at $10^{\circ} \mathrm{C}$ for 18 hours. Viral fraction was 198 collected with a syringe and stored in a 1:1 ratio with a storage buffer containing $10 \mathrm{mM}$ Tris, $\mathrm{pH}$ $1998.0,100 \mathrm{mM} \mathrm{NaCl}, 0.1$ percent BSA, and 50\% glycerol. HUVEC were treated with virus for 16 200 hours at a 1/10,000 final dilution in all cell culture experiments.

\section{Quantification of trafficking proteins.}


Quantification of pHlourin-podocalyxin was determined by projecting images (both 2D and $3 D$ cells/sprouts) in 3D using the FIJI XXX function and visually identifying accumulation(s) of pHlourin-podocalyxin relative to various plasma membranes. Quantification of WPB number was performed by counting VWF puncta on a per cell basis in both 2D culture and 3D sprouts. In both culture conditions, WPB localization was determined by first identifying WPB accumulations ( $\geq 4$ WPBs) or where the highest population resided in each individual cell. Second, images were

210 rendered as a 3D projection as mentioned above and the WPB puncta was scored based on its

211 most proximal membrane (e.g. basal or apical). Secretion of vWF was scored visually by 212 identifying whether vWF was in a lumen or contained within a cell. Actin stain determined cell and 213 lumen boundaries.

Immunofluorescence and Microscopy.

Prior to seeding cells, coverslips were treated with poly-D Lysine for approximately 20

217 minutes and washed 2 times with PBS. HUVECs were fixed with 4\% PFA for 7 minutes. ECs were

218 then washed three times with PBS and permeabilized with $0.5 \%$ Triton-X (Sigma) for 10 minutes.

219 After permeabilization, cells were washed three times with PBS. ECs were then blocked with $2 \%$ 220 bovine serum albumin (BSA) for 30 minutes. Once blocked, primary antibodies were incubated 221 for approximately 4-24 hours. Thereafter, primary antibodies were removed, and the cells were 222 washed 3 times with PBS. Secondary antibodies with 2\% BSA were added and incubated for 223 approximately 1-2 hours, washed 3 times with PBS and mounted on a slide for imaging. 
secondary antibody was added with $2 \%$ BSA and incubated overnight. Before imaging, the clot was washed 5 times with PBS. All primary and secondary antibodies are listed in the supplemental

232 data. Images were taken on a Nikon Eclipse Ti inverted microscope equipped with a CSU-X1

233 Yokogawa spinning disk field scanning confocal system and a Hamamatusu EM-CCD digital

234 camera. Images were captured using a Nikon Plan Apo 60x NA 1.40 oil objective using Olympus

235 type F immersion oil NA 1.518. All images were processed using ImageJ (FIJI).

\section{Statistical Analysis.}

Experiments were repeated a minimum of three times. Statistical analysis and graphing was performed using GraphPad Prism. Statistical significance was assessed with a Student's unpaired t-test for a two-group comparison. Statistical significance set a priori at $p<0.05$.

\section{RESULTS}

\section{Generating 3-dimensional sprouts using the fibrin-bead assay.}

To image endothelial-specific trafficking signatures we employed a fibrin-bead sprouting assay first described by Nakatsu et al. [20]. In this assay endothelial cells are coated onto a microcarrier bead and then embedded into a fibrin matrix. Following the addition of a fibroblast feeder

247 layer, the endothelial cells sprout into the surrounding matrix (Fig. 1A). Importantly, these sprouts 248 produce multicellular proto-vessel structures, as opposed to solely filopodia invasion such as 249 those observed in the Matrigel assay [21]. Here, sprouts reproduce characteristic in vivo sprouting 250 features such as branching, dynamic cell shuffling [22, 23], anastomosis [24], and lumen 251 formation [20] (Fig. 1B). There are other 3D sprouting assays that may demonstrate comparable 252 sprouting characteristics; however, in our hands, the fibrin-bead assay produced very distinct 253 multicellular sprouts with a clearly defined tip and stalk cell morphology. This is important, as other 254 assays can invade the surrounding matrix fashioning a cavernous lumen-like cavity, but this type 255 of morphology can be primarily attributed to cyst formation, where the cells breakdown the matrix, 
256 but lack canonical sprouting characteristics [25]. A possible drawback to this in vitro system is the

257 lack of blood flow-based morphodynamic cell rearrangements. Several groups have published

258 microfluidic devices that incorporate fluid-flow that could be potentially substituted here [26, 27].

259 Although, given the relative ease and low-cost of the fibrin-bead assay, it is likely more accessible

260 to the average laboratory. Additionally, many developmental trafficking events, such as those in

261 lumen biogenesis, precede blood flow, thus a 'flow-less' model would be appropriate in these

262 circumstances.

264 3D sprouts demonstrate a defined apical and basal polarity.

265 To determine the suitability of the fibrin-bead assay for trafficking studies we first examined

266 if sprout structures demonstrated apicobasal polarity as compared with 2D culture using standard

267 confocal imaging techniques. To test this, 3D sprouts generated in the fibrin-bead assay and

268 endothelial cells plated on coverslips (2D culture) were stained for actin (cytoskeleton), moesin

269 (apical), beta-1 integrin (basal), VE-cadherin (junctional) and podocalyxin (apical). 2D cultured

270 cells demonstrated a diffuse distribution of moesin and podocalyxin with no clear plasma

271 membrane enrichment, indicative of lack of apicobasal polarity (Fig. 2A). Orthogonal projections

272 could not provide useful information as the axial resolution was far too low to make out individual

273 puncta; therefore, we did not rely on this method going forward. In 2D, Beta-1 integrin was

274 localized to focal adhesions on the basal surface of the cell (Fig. 2A). One interesting note is that

275 endothelial cells cultured on non-compliant surfaces such as hard plastic or glass spread out to a

276 greater extent than cells cultured on soft matrices [28]. This elevated cell spreading in 2D could

277 also contribute to the decrease separation between apical and basal surfaces, complicating

278 discrete imaging of these membrane domains.

279 An immediately apparent advantage of the fibrin-bead system was that sprouts are

280 oriented in such a manner to the imaging plane that the apical and basal membranes are captured

281 in the $\mathrm{X}-\mathrm{Y}$ plane as opposed to $2 \mathrm{D}$ cells, where the apical and basal domains are in the $\mathrm{X}-\mathrm{Z}$ 
282 orientation (Fig. 2B). The X-Z axial plane has significantly lower resolution as compared to images

283 acquired in the X-Y plane. Using a conventional oil 1.4 NA 60x objective with a working distance

284 of $0.21 \mathrm{~mm}$, we encountered no issues imaging sprouts near the coverslip.

285 In contrast to 2D culture, fibrin-bead generated sprouts exhibited clearly polarized apical

286 and basal membrane surfaces. Sprouts stained for moesin showed a defined accumulation at the

287 apical membrane, distinct from VE-cadherin at cell-cell junctions and beta-1 integrin on the basal

288 membrane (Fig. 2B). Similarly, podocaylxin was highly enriched the apical membrane (Fig.

289 2B-D). We also observed that endothelial cells in sprouting structures displayed increased apical-

290 basal membrane separation as compared with cells cultured in 2D, this also enhanced our ability

291 to distinguish these domains (Fig. 2C,D). Overall, these results show fibrin-bead generated

292 sprouts demonstrate apical and basal signaling that can be imaged at high-resolution.

Dynamic Imaging of podocaylxin trafficking between 2D culture and 3D sprouting.

Podocalyxin is a glycoprotein that has been shown to be involved in lumen formation across many developmental models [14]. During lumen formation, podocalyxin is trafficked from the basal membrane to the apical membrane where it orchestrates delamination of the opposing cell membranes, creating the early lumen cavity [29-32]. Although many epithelial studies have documented podocalyxin's transcytosis, no endothelial studies have live-imaged this trafficking event to our knowledge. To image podocalyxin's insertion into the apical membrane we constructed a pHluorin-tagged podocalyxin adenovirus (Fig. 3A). PHluorin is a green fluorescent protein (GFP) variant that is non-fluorescent in acidified vesicles, but fluorescence is rescued at neutral $\mathrm{pH}$ following plasma membrane fusion (Fig. 3A) [33]. This approach allows us to 304 differentiate between Podxl that is inserted into the plasma membrane from populations that 305 reside in sub-apical vesicles. We first live-imaged the pHluroin-podocalyxin construct in 2D 306 cultured endothelial cells. Here, podocalyxin was localized to discrete puncta on both the apical 307 and basal domains of the cell (Fig. 3B,C; Movie 1); again, suggesting a lack of polarity. By 
contrast, live-imaging of pHluroin-podocalyxin in fibrin-bead assay sprouts demonstrated a robust and continuous accumulation of podocalyxin at the apical membrane with an elevated localization

310 to sites of lumen deadhesion (Fig. 3B,D; Movie 2). In line with previous reports, our results

311 demonstrate that podocalyxin is actively trafficked to the apical membrane; however, it may be

312 trafficked more robustly to cell-cell interfaces actively undergoing membrane deadhesion via

313 undescribed mechanisms. These data also indicate that sub-cellular podocalyxin trafficking

314 events can be captured at high-resolution in the fibrin-bead sprouting assay.

316 Characterizing Rab35 trafficking between 2D culture and 3D sprouting.

317 Rab GTPases represent a class of well-studied proteins that orchestrate vesicle trafficking

$318[6,34,35]$. In particular, Rab35 has been shown to play a multitude of roles depending on the

319 organism, tissue-type and cellular conditions [36-39]. Also, Rab35's localization has not been

320 characterized in vascular tissue. Thus, to explore the use of the fibrin-bead assay for imaging

321 endothelial trafficking events, we over-expressed Rab35 in both 2D and 3D culture conditions. In

322 2D culture, GFP-Rab35 displayed a membranous localization but was broadly distributed, not co-

323 localizing with basal marker Beta-1 integrin, nor cytoskeletal protein actin (Fig. 4A,B). By contrast,

324 expression of GFP-Rab35 in 3D fibrin-bead sprouts demonstrated a preference for the apical

325 membrane co-localizing with actin and distinct from beta1 integrin (Fig. 4C,D).

326 Live-imaging in 2D culture showed GFP-Rab35 localized to dorsal membrane protrusions

327 and lamellipodia; however, there was no membrane bias that was readily apparent (Fig. 5A;

328 Movie 3). Live-imaging of Rab35 in the 3D sprouts revealed that, again, Rab35 is membranous

329 with a preference to the apical membrane (Fig. 5B). Additionally, unique to the 3D sprouting

330 environment, we could observe individual endosome movements adjacent to the apical

331 membrane, which we could not detect in 2D culture (Fig. 5B; Movie 4). Interestingly, compared

332 with 2D culture, the membrane dynamics were vastly dampened in 3D sprouting, suggesting

333 culture in 2D may increase membrane dynamics consistent with other reports [40]. Overall, this 
334 data not only demonstrates a clear difference between Rab35 trafficking in 2D and 3D culture

335 systems, but shows the importance of fully resolving apical and basal domains to interrogate

336 function.

338 Capturing exocytic events between 2D culture and 3D sprouting.

An endothelial-specific trafficking function is the secretion of clotting proteins such as von

340 Willebrand Factor into the luminal space upon injury [41-43]. WPBs are cigar shaped vesicles that

341 are rapidly deployed to the apical membrane to exocytose many components including pro-

342 thrombotic vWF [43]. We sought to determine if tracking WPB-related secretion events in 3D

343 sprouts was feasible. We believe this is important as the majority of reports examining WPB

344 biology use 2D culture opposed to tracking exocytic events in 3D sprouts with a more

345 physiological defined apicobasal polarity. To do so, in 2D culture, we expressed GFP-Rab27a

346 which has been previously shown to decorate WPBs [44-46]. Consistent with other reports

347 Rab27a strongly co-localized with vWF on WPB puncta [44-46]. Co-labeled WPB puncta were

348 randomly distributed throughout the cell with no distinguishable membrane preference (Fig. 6A).

349 In 3D fibrin-bead sprouts Rab27a and vWF co-stained WPBs were easily visualized in proximity

350 to apical or basal domains. In accordance with WPB function, we observed that WPBs

351 accumulated at the apical membrane in many instances (Fig. 6B). Interestingly, we observed that

352 WPB number is generally increased in ECs within sprout structures as compared with ECs in 2D

353 culture (Fig. 6C). Of the WPBs in 2D culture there was almost equal split between the number of

354 WPB accumulations that were in close proximity to the basal domain ( $\leq 1 \mathrm{um})$ and generally

355 contained within the body of cytoplasm, with $10 \%$ of WPBs at the apical domain (Fig. 6D). By

356 contrast ECs in a 3D sprout showed that $36 \%$ of WPB accumulations were near the apical domain,

$35750 \%$ in the cytoplasm, $0 \%$ at the basal domain with the remaining $17 \%$ exocytosed into the lumen

358 cavity (Fig. 6D). These results demonstrate that WPBs can be resolved 3D sprouts. 
Moving to live-imaging of WPBs labeled with Rab27a in 2D, we easily resolve discrete puncta and the disappearance of individual WPBs, presumably being exocytosed (Fig. 7A; Movie 5). In live-imaged 3D sprouts prior to lumen formation, we observed a robust accumulation of

362 WPBs at the cell-cell interface as previously described by our group (Fig. 7B; Movie 6) [44].

363 Unlike 2D culture, we resolved WPBs aggregated at the apical membrane with less WPBs near

364 the basal surface and were able to temporospatially track these structures relative to membrane

365 domains (Fig. 7B). These results demonstrate that in the fibrin-bead sprouts sub-cellular WPB

366 puncta can be tracked with enough resolution that monitoring movements between apical and

367 basal surfaces can be achieved. This is an important aspect as exocytosis of WPB components

368 will occur at the apical membrane.

Next, we compared exocytosis of WPB-housed vWF between 2D culture and fibrin-bead sprouts. The rationale for this was: 1) the primary function of vWF is to be secreted, thus we wanted to capture this process in a 3D multicellular sprout structure; and 2) for a direct comparison

372 to the standard 2D exocytosis assay of vWF many others have employed [10, 46, 47]. In 2D

373 culture we knocked down Rab27a which has been shown to induce tonic vWF secretion [10]. In

374 the Rab27a knockdown cells there was qualitatively less vWF puncta as compared with the 375 scrambled controls; although, the amount of VWF exocytosis could not be assessed by imaging 376 alone as it was secreted directly into the surrounding media (Fig. 8A,C). By contrast, Rab27a 377 knockdown in fibrin-bead sprouts showed a dramatic accumulation of vWF effectively trapped 378 within the sprout lumen (Fig. 8B). We could reproduce this result by administration of ionomycin 379 (Fig. 8D,E), thus providing a conditional aspect to WPB evoked secretion. We believe this offers 380 advantages over 2D culture in providing a better capacity to capture secretion events directed at 381 the apical membrane in a growing sprout while simultaneously visualizing the relative amount of 382 secreted protein(s) in the luminal space. These results show the utility of using a 3D sprouting 383 culture system for visualizing exocytic events. 


\section{DISCUSSION}

In the current investigation we show that the fibrin-bead sprouting assay has tremendous potential for imaging subcellular trafficking events. A primary disadvantage of in vivo imaging is the inability to capture trafficking processes due to the relative incompatibility of penetrating tissue with a long-working distance objective while maintaining high-enough resolution to resolve subcellular processes. Additionally, in many in vivo models, capturing dynamic events at the spatial scales required to distinguish trafficking processes, is not feasible. A common workaround to

392 these issues is 2D culture of endothelial cells. However, our work establishes that this type of 393 culture system strips away apicobasal signaling that is paramount to many trafficking processes.

394 Our results using the fibrin-bead model highlights a middle ground where 3D sprouts that embody 395 the most salient physiological characteristics of in vivo sprouting can be imaged at high-resolution on common confocal microscope platforms. Importantly, we demonstrate that imaging 3D sprouts not only presents advantages in capturing apical and basal domains due to sprout orientation, but is required to initiate proper apicobasal polarity. In the fibrin-bead we could clearly resolve trafficking mediators Rab35 and Rab27a both in live and fixed specimens and show their differential localization to the apical membrane. We also demonstrate the enhanced utility of the fibrin-bead assay for imaging exocytic proteins that are sequestered in the luminal cavity. Overall,

402 our results support that the fibrin-bead sprouting model is suitable for visualizing endothelial 403 trafficking events and could serve as an excellent companion assay to in vivo work.

405 how trafficking, such as those in lumen biogenesis or secretion, are best imaged in 3D sprout 406 structures. By virtue of being able to capture a cross-section of the sprout long-axis, imaging 407 apical and basal domains is very accessible as this is the natural X-Y plane, unless acquired by 408 lightsheet microscopy. Conversely, the apicobasal orthogonal view in cells imaged on a 2D 409 surface needs to be digitally reconstructed limiting resolution. A problem that we originally 410 envisioned was that the sprouts themselves would be too far from the coverslip limiting use of 
411 high numerical aperture, low working distance objectives. This was not the case, as most sprouts

412 grew well within the working distance of a non-long working distance 60x objective. Static imaging

413 of cell polarity markers provided a strong indication that virtually no apical signaling is present in

$4142 \mathrm{D}$ culture as compared with 3D sprouts as luminal proteins moesin and podocalyxin did not

415 localize to the apical domain. Depending on the experimental question, this may not be a major

416 issue. For instance, imaging of cytoskeletal proteins, this would likely not pose a problem.

417 However, an issue arises when testing processes that require apicobasal polarity that is otherwise

418 non-existent in 2D culture. This lack of polarity was also obvious when imaging Rab35 as it

419 demonstrated apical localization in 3D sprouts and somewhat random localization on membrane

420 protrusions in 2D cells.

$421 \quad$ A surprising finding was that exocytosis of vWF was perfectly contained within the luminal

422 cavity when using the fibrin-bead assay. Imaging individual sprouts, we could very easily resolve

423 discrete WPBs adjacent to the apical membrane, but could also potentially quantify the relative

424 exocytosis of proteins on a per sprout basis given the secreted proteins are confined to the lumen

425 cavity. In 2D culture, secreted proteins diffuse into the surrounding media blocking the ability to

426 ascribe local secretion events to a particular group of cells. Given the preponderance of literature

427 investigating WPB trafficking function using 2D culture, the fibrin-bead assay may be very

428 beneficial in providing a more physiological component to these studies.

429 Culturing endothelial cells in 3D matrixes to induce sprouting behaviors is not new to the

430 field of angiogenesis. Assays such as the Matrigel [21], vasculogenic assay [30, 48, 49], hanging-

431 drop [50, 51], and fibrin-bead methods were initially used to investigate differences in endothelial

432 sprouting and branching characteristics. For these types of gross morphometric analyses, these

433 assays are still widely used; although, there are major advantages and disadvantages to each

434 method. What has changed in more recent years is a focus on cell autonomous molecular

435 mechanisms of sprouting angiogenesis that require an ever expanding need to visualize sub-

436 cellular processes. In this regard, our group heavily employs the fibrin-bead assay, because as 
437 we focused on trafficking processes, we found a tremendous difference in 3D sprouting vs 2D

438 culture systems. The differences in signaling between 2D plated cells and 3D sprouts was

439 extreme enough to mask group changes in studies using loss and gain of function approaches.

440 Not detecting differences makes sense if the 2D culture system does not provide apicobasal

441 signaling cues, then perturbations in this polarity axis will not be readily apparent and lost to the

442 investigator.

443 Our endorsement of the fibrin-bead sprouting assay does not exclude the possibility of

444 other in vitro angiogenic or vasculogenic assays for imaging trafficking events as well as some in

445 vivo models. For example, Davis et al. has demonstrated the excellent utility of the vasculogenic

446 endothelial cell assay for not only tracking sprouting parameters, but visualizing sub-cellular

447 processes like cytoskeletal proteins and caveolin localization [48, 52, 53]. Likewise, the original

448 group who created the fibrin-bead assay has also engineered a microfluidic sprouting system that

449 could be very interesting for investigating trafficking patterns with flow [27]. For in vivo models,

450 unlike mammalians, zebrafish are optically transparent early in development allowing for live-

451 imaging of blood vessel processes [54-57]. Many sub-cellular structures in endothelial cells can

452 be distinguished using this model; however, in our hands, we cannot yield the resolution required

453 to confidently quantify subtle trafficking localization events using needed $20 x$ and $40 x$ long

454 working distance objectives. Therefore, we believe having the fibrin-bead assay to complement

455 an in vivo model can help bridge deficits in either approach. There are many others who have

456 developed comparable blood vessel sprouting-related assays that may be suited for imaging

457 trafficking events that we have not mentioned. Our primary aim in the current investigation was to

458 report our overwhelmingly positive experience with the fibrin-bead assay for imaging trafficking-

459 related programs during angiogenic sprouting.

460 In conclusion, our data demonstrates that the fibrin-bead sprouting assay is an excellent

461 platform for imaging endothelial trafficking events, particularly those related to apical and basal

462 domains. We believe this method is a good substitute for 2D culture on many levels, most notably 
463 for imaging endothelial-related trafficking behaviors. We believe that endothelial-specific

464 trafficking signatures represent a novel level of regulation that significantly contributes to vascular

465 form and function. Thus, tools that aid in characterizing these processes will allow researchers to

466 answer novel questions related to endothelial biology.

467

468 SOURCES OF FUNDING

469 Work was supported by funding from the National Heart Lung Blood Institute (Grant

470 1R56HL148450-01, R00HL124311) (E.J.K).

471

472 CONTRIBUTIONS

473 C.R.F performed all experiments. C.R.F and E.J.K wrote the manuscript.

475 DISCLOSURES

$476 \quad$ None 


\section{REFERENCES}

480 1. Bussmann, J., S.A. Wolfe, and A.F. Siekmann, Arterial-venous network formation during brain vascularization involves hemodynamic regulation of chemokine signaling. Development, 2011. 138(9): p. 1717-26.

2. Kushner, E.J. and V.L. Bautch, Building blood vessels in development and disease. Curr Opin Hematol, 2013. 20(3): p. 231-6.

3. Farquhar, M.G., Multiple pathways of exocytosis, endocytosis, and membrane recycling: validation of a Golgi route. Fed Proc, 1983. 42(8): p. 2407-13.

4. Orzech, E., et al., Interactions between the exocytic and endocytic pathways in polarized Madin-Darby canine kidney cells. J Biol Chem, 2000. 275(20): p. 15207-19.

5. Foley, K., S. Boguslavsky, and A. Klip, Endocytosis, recycling, and regulated exocytosis of glucose transporter 4. Biochemistry, 2011. 50(15): p. 3048-61. therapeutic opportunities. Clin Genet, 2011. 80(4): p. 305-18.
6. Agola, J.O., et al., Rab GTPases as regulators of endocytosis, targets of disease and
7. Hutagalung, A.H. and P.J. Novick, Role of Rab GTPases in membrane traffic and cell physiology. Physiol Rev, 2011. 91(1): p. 119-49.

8. Segev, N., GTPases in intracellular trafficking: an overview. Semin Cell Dev Biol, 2011. 22(1): p. 1-2.

9. Mizuno-Yamasaki, E., F. Rivera-Molina, and P. Novick, GTPase networks in membrane traffic. Annu Rev Biochem, 2012. 81: p. 637-59.

500

10. Bierings, R., et al., The interplay between the Rab27A effectors SIp4-a and MyRIP controls hormone-evoked Weibel-Palade body exocytosis. Blood, 2012. 120(13): p. 2757-67.

501

502

503

504

505

506

507

508
11. Jovic, M., et al., The early endosome: a busy sorting station for proteins at the crossroads. Histol Histopathol, 2010. 25(1): p. 99-112.

12. Tian, T., et al., Dynamics of exosome internalization and trafficking. J Cell Physiol, 2013. 228(7): p. 1487-95.

13. Weisz, O.A. and E. Rodriguez-Boulan, Apical trafficking in epithelial cells: signals, clusters and motors. J Cell Sci, 2009. 122(Pt 23): p. 4253-66.

14. Mrozowska, P.S. and M. Fukuda, Regulation of podocalyxin trafficking by Rab small GTPases in 2D and 3D epithelial cell cultures. J Cell Biol, 2016. 213(3): p. 355-69.

15. Dudley, A.C., Tumor endothelial cells. Cold Spring Harb Perspect Med, 2012. 2(3): p. a006536.

16. Nakatsu, M.N., J. Davis, and C.C. Hughes, Optimized fibrin gel bead assay for the study of angiogenesis. J Vis Exp., 2007(3): p. 186. doi: 10.3791/186. Epub 2007 Apr 29.

17. Campeau, E., et al., A versatile viral system for expression and depletion of proteins in mammalian cells. PLoS One, 2009. 4(8): p. e6529.

18. Gibson, D.G., et al., Enzymatic assembly of DNA molecules up to several hundred kilobases. Nat Methods, 2009. 6(5): p. 343-5.

19. He, T.C., et al., A simplified system for generating recombinant adenoviruses. Proc Natl Acad Sci U S A, 1998. 95(5): p. 2509-14.

20. Nakatsu, M.N., et al., Angiogenic sprouting and capillary lumen formation modeled by human umbilical vein endothelial cells (HUVEC) in fibrin gels: the role of fibroblasts and Angiopoietin-1. Microvasc Res, 2003. 66(2): p. 102-12.

21. Crabtree, B. and V. Subramanian, Behavior of endothelial cells on Matrigel and development of a method for a rapid and reproducible in vitro angiogenesis assay. In Vitro Cell Dev Biol Anim, 2007. 43(2): p. 87-94.

22. Kushner, E.J., et al., Excess Centrosomes Perturb Dynamic Endothelial Cell Repolarization During Blood Vessel Formation. Mol Biol Cell, 2016.

23. Jakobsson, L., et al., Endothelial cells dynamically compete for the tip cell position during angiogenic sprouting. Nat Cell Biol, 2010. 12(10): p. 943-53. 
562

563

564

565

566

567

568

569

570

571

572

573

574

575

576

577

578

24. Nesmith, J.E., et al., Blood vessel anastomosis is spatially regulated by Flt1 during angiogenesis. Development, 2017. 144(5): p. 889-896.

25. Dubois-Stringfellow, N., A. Jonczyk, and V.L. Bautch, Perturbations in the fibrinolytic pathway abolish cyst formation but not capillary-like organization of cultured murine endothelial cells. Blood, 1994. 83(11): p. 3206-17.

26. Wang, X., et al., Engineering anastomosis between living capillary networks and endothelial cell-lined microfluidic channels. Lab Chip, 2016. 16(2): p. 282-90.

27. Sobrino, A., et al., 3D microtumors in vitro supported by perfused vascular networks. Sci Rep, 2016. 6: p. 31589.

28. Engler, A., et al., Substrate compliance versus ligand density in cell on gel responses. Biophys J., 2004. 86(1 Pt 1): p. 617-28.

29. Bryant, D.M., et al., A molecular switch for the orientation of epithelial cell polarization. Dev Cell, 2014. 31(2): p. 171-87.

30. Davis, G.E. and O.B. Cleaver, Outside in: inversion of cell polarity controls epithelial lumen formation. Dev Cell, 2014. 31(2): p. 140-2.

31. Meder, D., et al., Gp135/podocalyxin and NHERF-2 participate in the formation of a preapical domain during polarization of MDCK cells. J Cell Biol., 2005. 168(2): p. 303-13. Epub 2005 Jan 10.

32. Yasuda, T., et al., Rab27 effector SIp2-a transports the apical signaling molecule podocalyxin to the apical surface of MDCK II cells and regulates claudin-2 expression. Mol Biol Cell, 2012. 23(16): p. 3229-39.

33. Sankaranarayanan, S., et al., The use of pHluorins for optical measurements of presynaptic activity. Biophys J, 2000. 79(4): p. 2199-208.

34. Bryant, D.M., et al., A molecular network for de novo generation of the apical surface and lumen. Nat Cell Biol, 2010. 12(11): p. 1035-45.

35. Grosshans, B.L., D. Ortiz, and P. Novick, Rabs and their effectors: achieving specificity in membrane traffic. Proc Natl Acad Sci U S A, 2006. 103(32): p. 11821-7.

36. Allaire, P.D., et al., The Connecdenn DENN domain: a GEF for Rab35 mediating cargospecific exit from early endosomes. Mol Cell, 2010. 37(3): p. 370-82.

37. Klinkert, K. and A. Echard, Rab35 GTPase: A Central Regulator of Phosphoinositides and F-actin in Endocytic Recycling and Beyond. Traffic, 2016. 17(10): p. 1063-77.

38. Shaughnessy, R. and A. Echard, Rab35 GTPase and cancer: Linking membrane trafficking to tumorigenesis. Traffic, 2018. 19(4): p. 247-252.

39. Villagomez, F.R., et al., The role of the oncogenic Rab35 in cancer invasion, metastasis, and immune evasion, especially in leukemia. Small GTPases, 2018: p. 1-12.

40. Gardel, M.L., et al., Mechanical integration of actin and adhesion dynamics in cell migration. Annu Rev Cell Dev Biol, 2010. 26: p. 315-33.

41. Hattori, R., et al., Stimulated secretion of endothelial von Willebrand factor is accompanied by rapid redistribution to the cell surface of the intracellular granule membrane protein GMP-140. J Biol Chem, 1989. 264(14): p. 7768-71.

42. Lillicrap, D., von Willebrand disease: advances in pathogenetic understanding, diagnosis, and therapy. Blood, 2013. 122(23): p. 3735-40.

43. Nightingale, T.D., et al., Tuning the endothelial response: differential release of exocytic cargos from Weibel-Palade bodies. J Thromb Haemost, 2018. 16(9): p. 1873-1886.

44. Francis, C.R., S. Claflin, and E.J. Kushner, Synaptotagmin-Like Protein 2a Regulates Angiogenic Lumen Formation via Weibel-Palade Body Apical Secretion of Angiopoietin-2. Arterioscler Thromb Vasc Biol, 2021: p. Atvbaha121316113.

45. Schillemans, M., et al., Weibel-Palade Body Localized Syntaxin-3 Modulates Von Willebrand Factor Secretion From Endothelial Cells. Arterioscler Thromb Vasc Biol, 2018. 38(7): p. 1549-1561. 
46. van Breevoort, D., et al., STXBP1 promotes Weibel-Palade body exocytosis through its interaction with the Rab27A effector Slp4-a. Blood, 2014. 123(20): p. 3185-94.

47. Lenzi, C., et al., Synaptotagmin 5 regulates Ca(2+)-dependent Weibel-Palade body exocytosis in human endothelial cells. J Cell Sci, 2019. 132(5).

48. Davis, G.E., K.J. Bayless, and A. Mavila, Molecular basis of endothelial cell morphogenesis in three-dimensional extracellular matrices. Anat Rec., 2002. 268(3): p. 252-75.

49. Davis, G.E. and D.R. Senger, Endothelial extracellular matrix: biosynthesis, remodeling, and functions during vascular morphogenesis and neovessel stabilization. Circ Res, 2005. 97(11): p. 1093-107.

50. Tetzlaff, F. and A. Fischer, Human Endothelial Cell Spheroid-based Sprouting Angiogenesis Assay in Collagen. Bio-protocol, 2018. 8(17): p. e2995.

51. Timmins, N.E., S. Dietmair, and L.K. Nielsen, Hanging-drop multicellular spheroids as a model of tumour angiogenesis. Angiogenesis, 2004. 7(2): p. 97-103.

52. Norden, P.R., et al., Cdc42 and k-Ras Control Endothelial Tubulogenesis through Apical Membrane and Cytoskeletal Polarization: Novel Stimulatory Roles for GTPase Effectors, the Small GTPases, Rac2 and Rap1b, and Inhibitory Influence of Arhgap31 and Rasa1. PLoS One, 2016. 11(1): p. e0147758.

53. Norden, P.R., Z. Sun, and G.E. Davis, Control of endothelial tubulogenesis by Rab and Ral GTPases, and apical targeting of caveolin-1-labeled vacuoles. PLoS One, 2020. 15(6): p. e0235116.

54. Akerberg, A.A., S. Stewart, and K. Stankunas, Spatial and temporal control of transgene expression in zebrafish. PLoS One, 2014. 9(3): p. e92217.

55. Gore, A.V., et al., Vascular development in the zebrafish. Cold Spring Harb Perspect Med, 2012. 2(5): p. a006684.

56. Gut, P., et al., LITTLE FISH, BIG DATA: ZEBRAFISH AS A MODEL FOR CARDIOVASCULAR AND METABOLIC DISEASE. Physiol Rev, 2017. 97(3): p. 889-938.

57. Lawson, N.D. and B.M. Weinstein, In vivo imaging of embryonic vascular development using transgenic zebrafish. Dev Biol., 2002. 248(2): p. 307-18. 


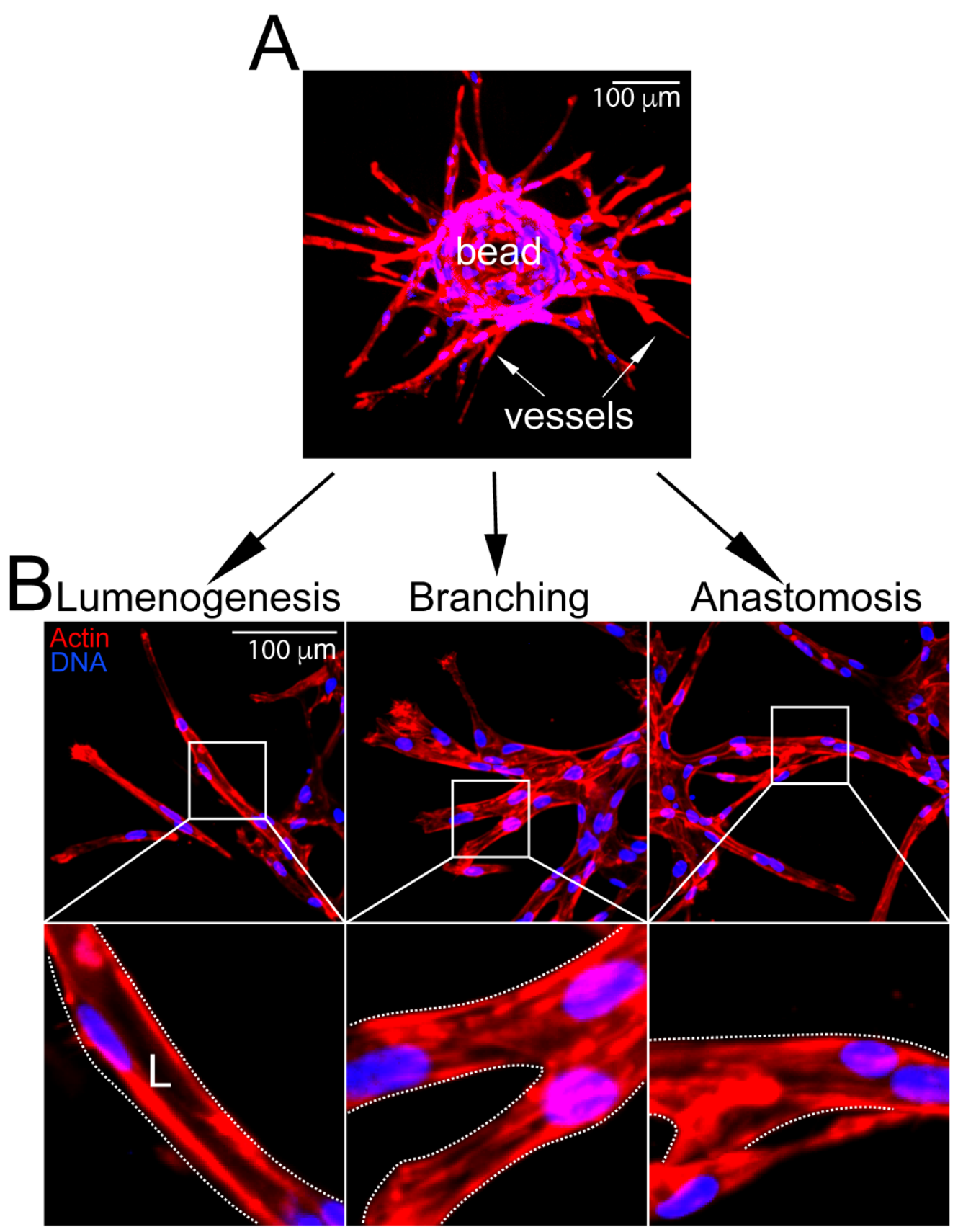

Figure 1. Fibrin-bead assay recapitulates angiogenic traits in vitro. Top- representative image of embedded fibrin-bead after 4 days of growth. Bottom- representative images of lumenogenesis, branching and anastomosis with magnifications (boxes). $L$ denotes lumen. 


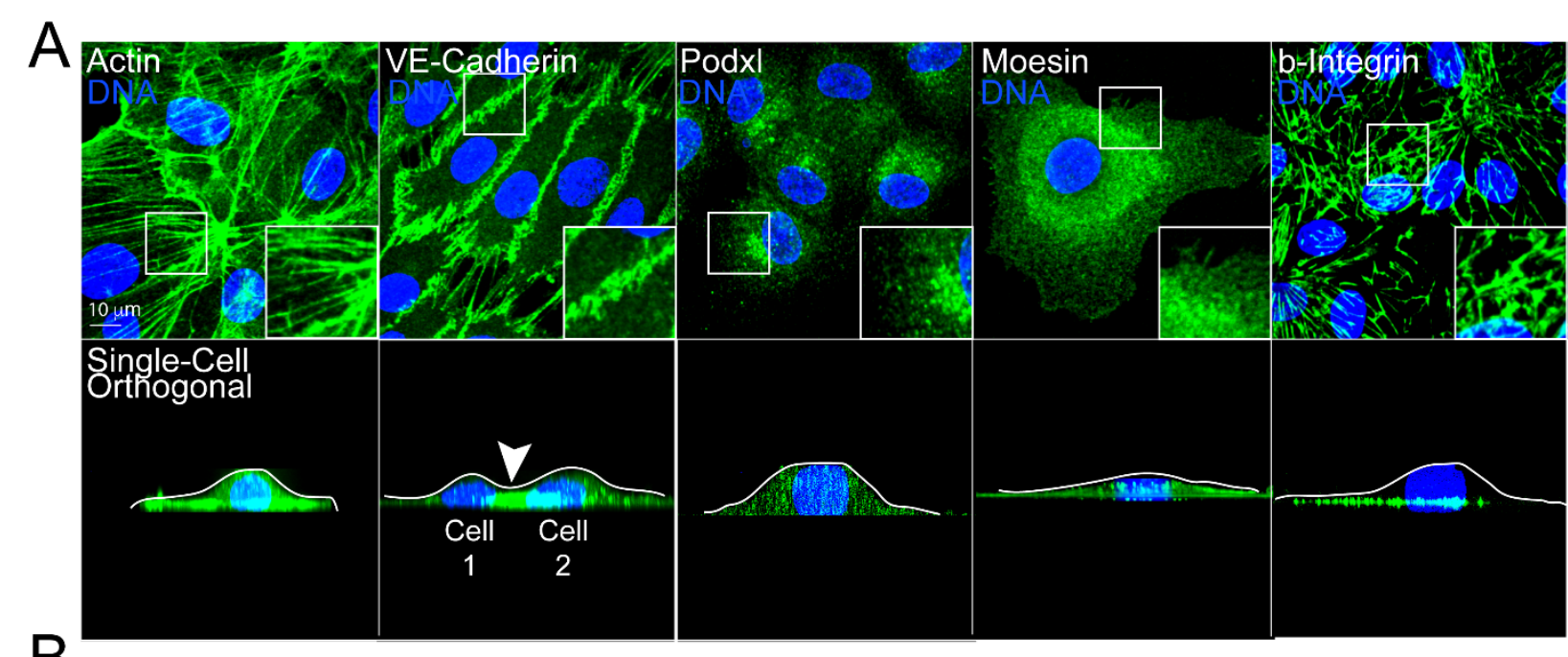

B
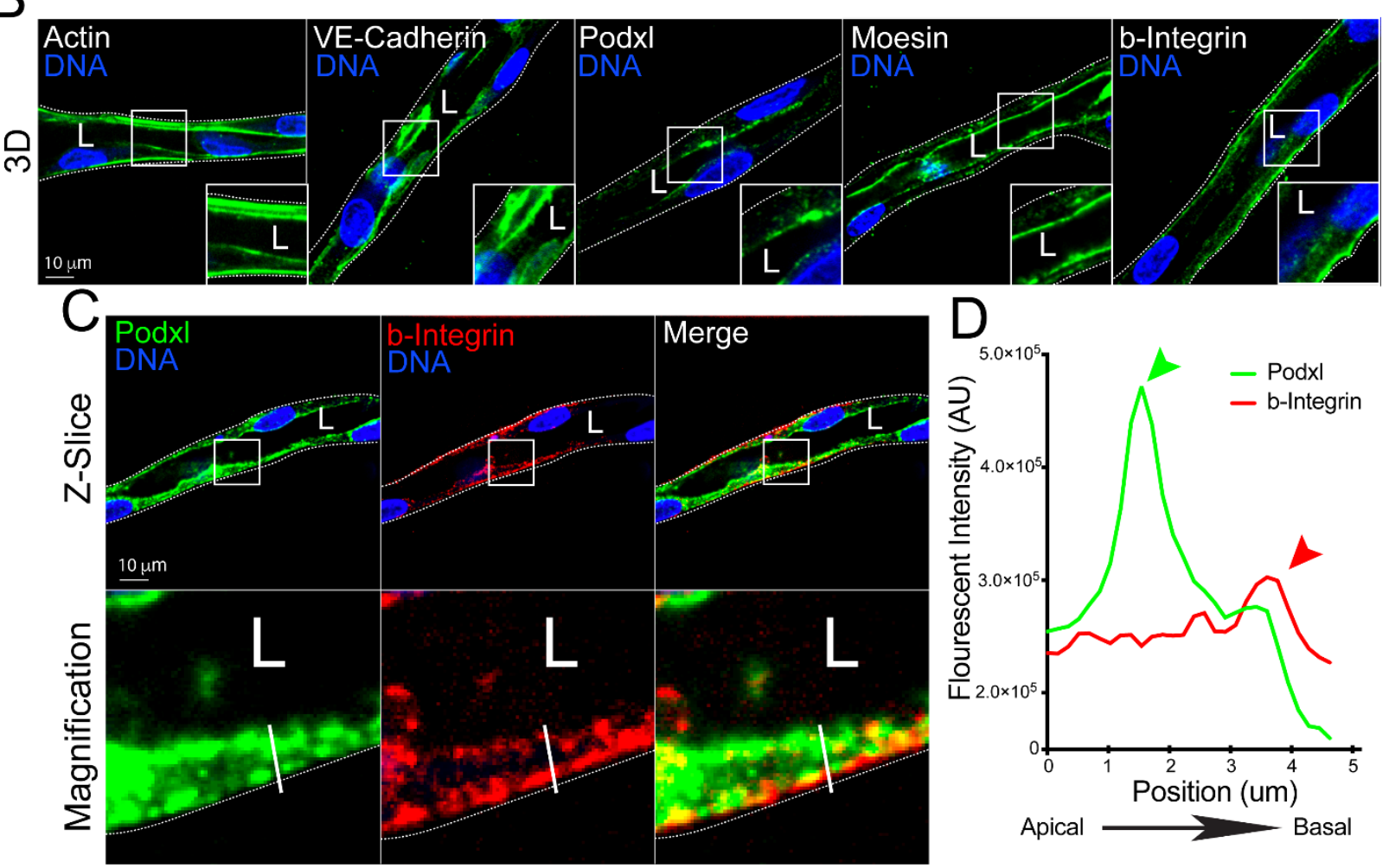

Figure 2. Comparison of cell polarity markers in 2D culture and 3D sprouts. (A) Representative images of endothelial cell cultured on 2D surface (top panels) and axial view (x-Z plane, bottom panels). Cells were stained with actin (cytoskeleton), VE-cadherin (cell junctions), podocalyxin (Podxl, apical membrane), moesin (apical membrane), and beta-1 integrin (bintegrin, basal membrane). White lines mark apical surface and arrowhead denotes junction between two cells. (B) Representative images of fibrin-bead generated sprouts stained for indicated proteins. (C) Representative imaging showing co-staining of Podxl and b-integrin within the same sprout cross-section to highlight differences in apical and basal domains. (D) Line scan illustrating peaks in fluorescent intensity of podxl and b-Integrin relative to apical and basal domains. The green arrowhead denotes peak of podxl and red arrowhead denotes peak of bIntegrin. The white line in panel $\mathrm{C}$ denotes the line scan area. White boxes are areas of 


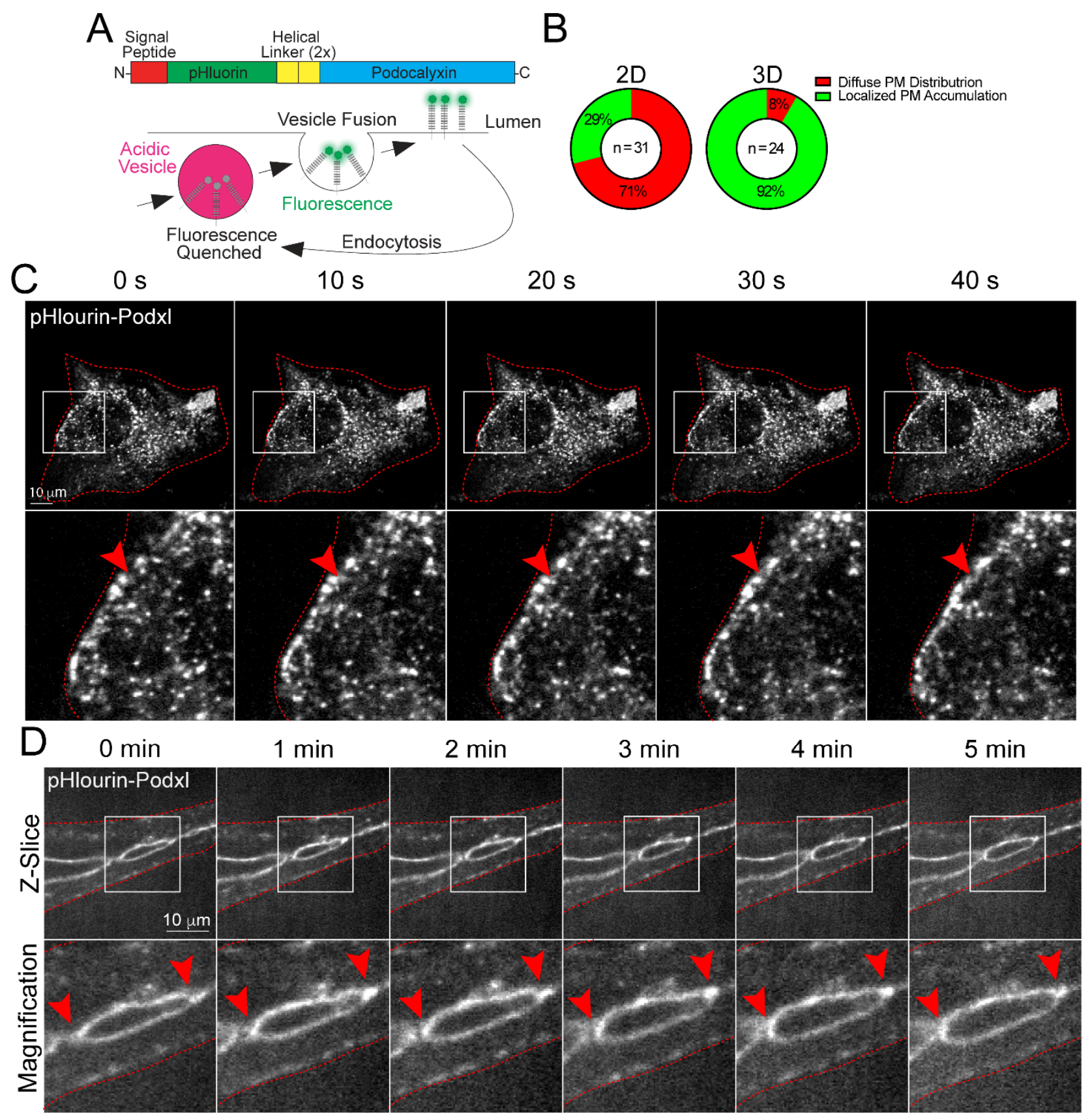

Figure 3. Live-imaging of podocalyxin trafficking in 2D culture and 3D sprouts. (A) Structure of engineered pHluorin-Podxl fusion protein. In acidified vesicles, pHluorin fluorescence is significantly quenched. However, once inserted on the plasma membrane at neutral $\mathrm{pH}$, fluorescence is rescued allowing for visualization of plasma membrane insertion. (B) Graph showing percentages of pHluorin-Podxl with either even or punctate plasma membrane (PM) distribution. (C) Live-imaging of 2D cell expressing pHluorin-Podxl over time. Red arrowheads denote puncta accumulated at the leading edge of the cell. (D) Live-imaging of fibrin-bead generated sprout expressing pHluorin-Podxl over time. Red arrowheads indicate active areas of lumen expansion where Podxl is accumulating. White boxes are areas of magnification and red 

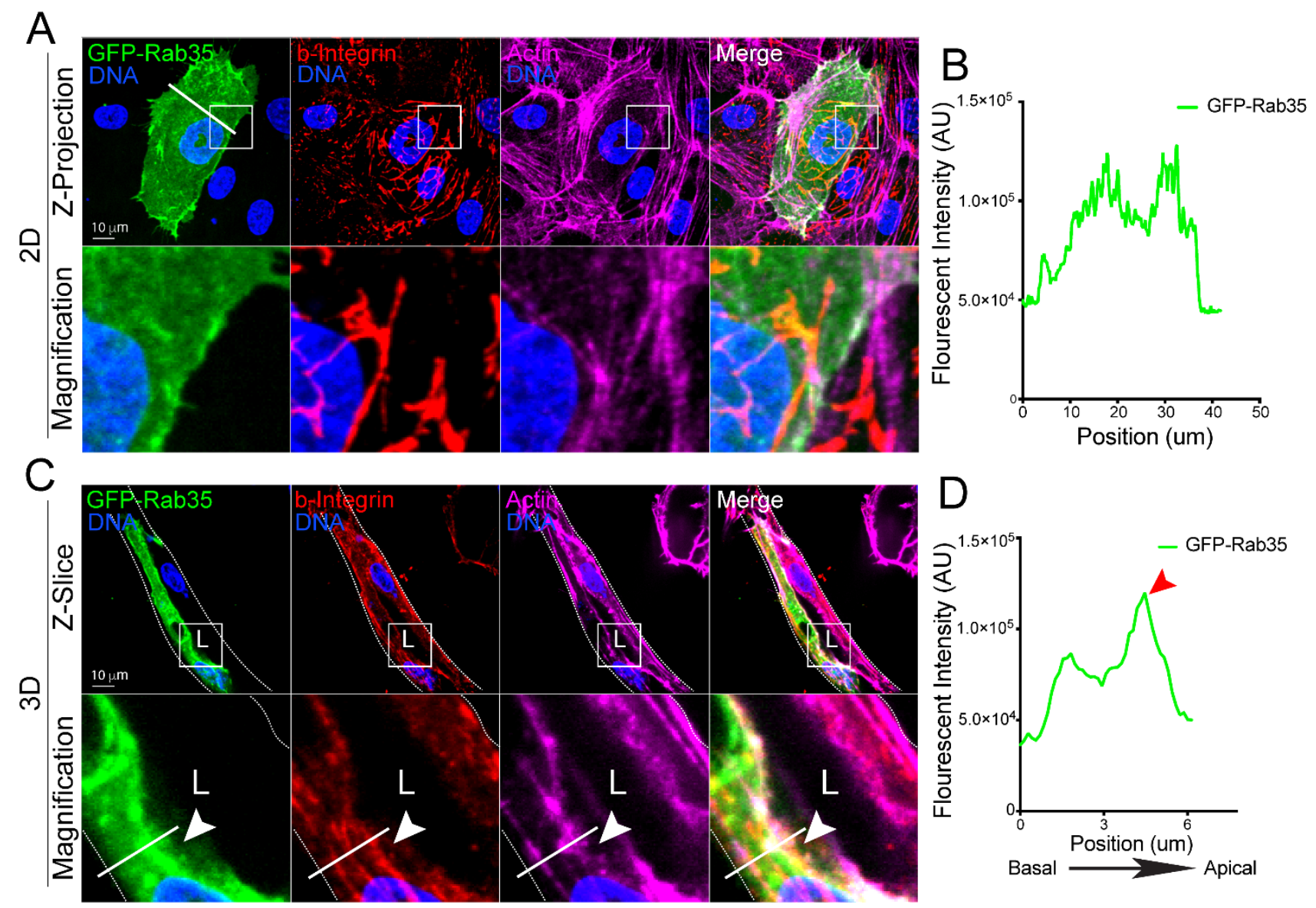

Figure 4. Visualizing Rab35 GTPase localization in 2D culture and 3D sprouts. (A) Representative image of endothelial cell expressing GFP-Rab35, stained for beta-1 integrin (bintegrin) and actin in 2D. Lower panels are magnification. (B) Line scan of Rab35 intensity in panel A. White line across cell in panel A represents line scan location. (C) Representative image of fibrin-bead sprout expressing GFP-Rab35, stained for b-integrin and actin. (D) Line scan of Rab35 intensity in panel C. White boxes are areas of magnification and while dotted lines indicated sprout boundaries. $L$ denotes lumen. 


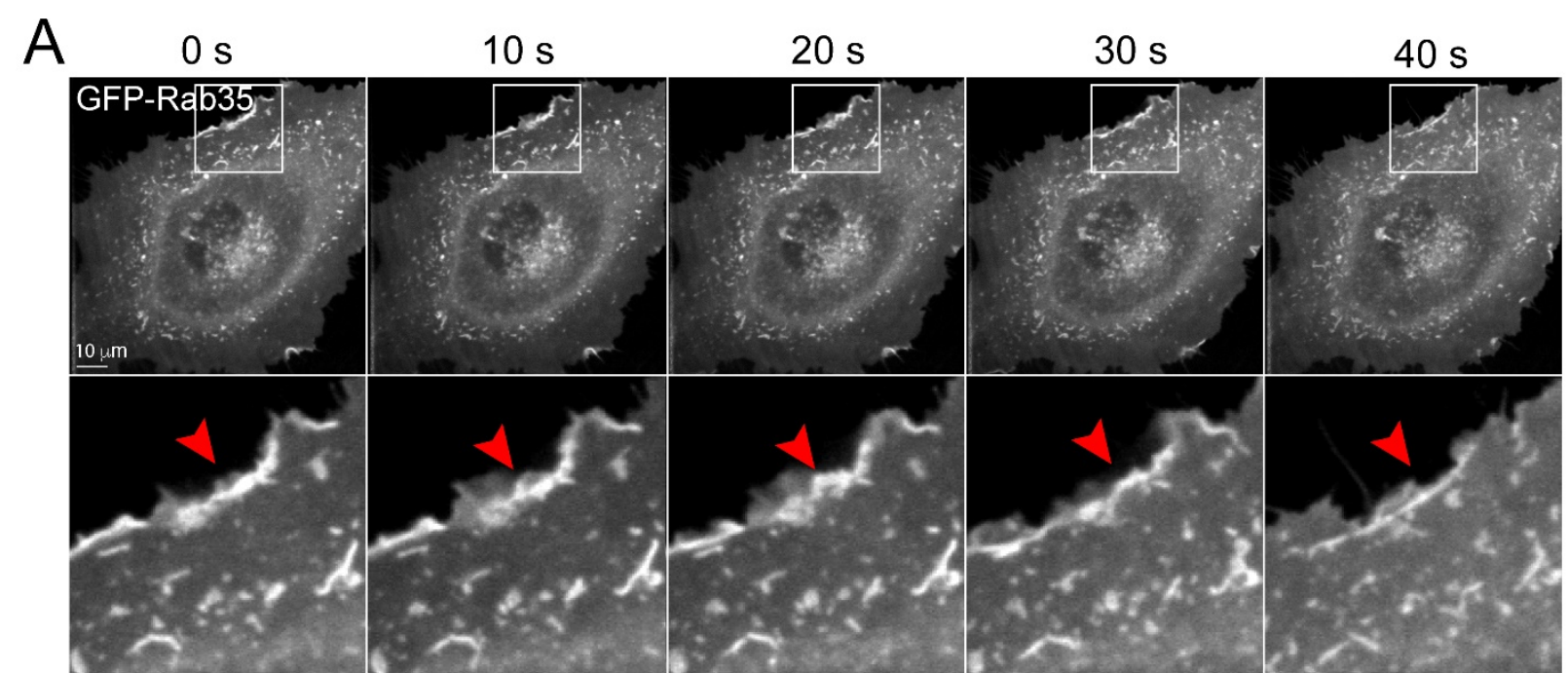

B

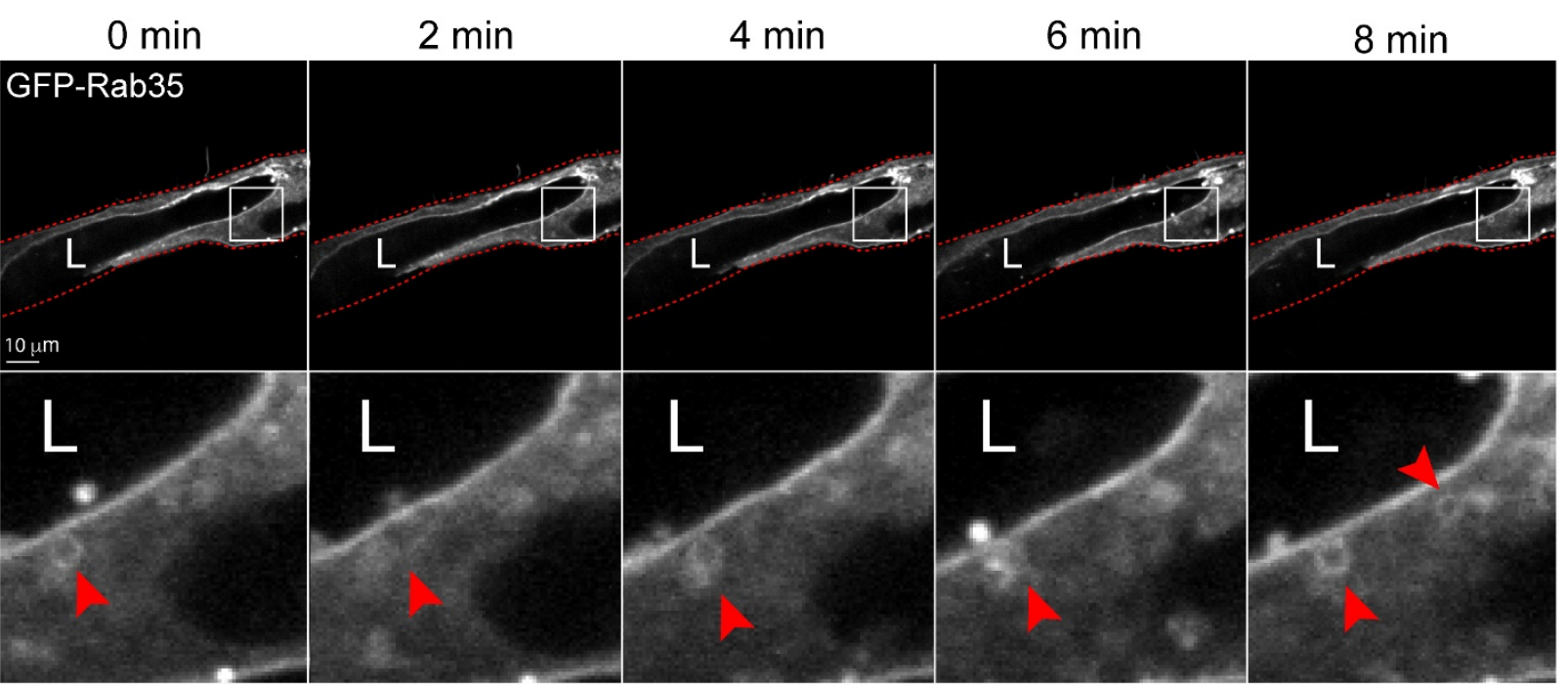

680
Figure 5. Live-imaging of Rab35 trafficking in 2D culture and 3D sprouts. (A) Live-imaging of cell expressing GFP-Rab35 over time. Red arrowheads denote puncta accumulated at the leading edge of the cell. (B) Live-imaging of fibrin-bead generated sprout expressing GFP-Rab35 over time. Red arrowheads indicate small endosome movements. White boxes are areas of magnification and red lines indicated sprout boundaries. $L$ denotes lumen. 
699

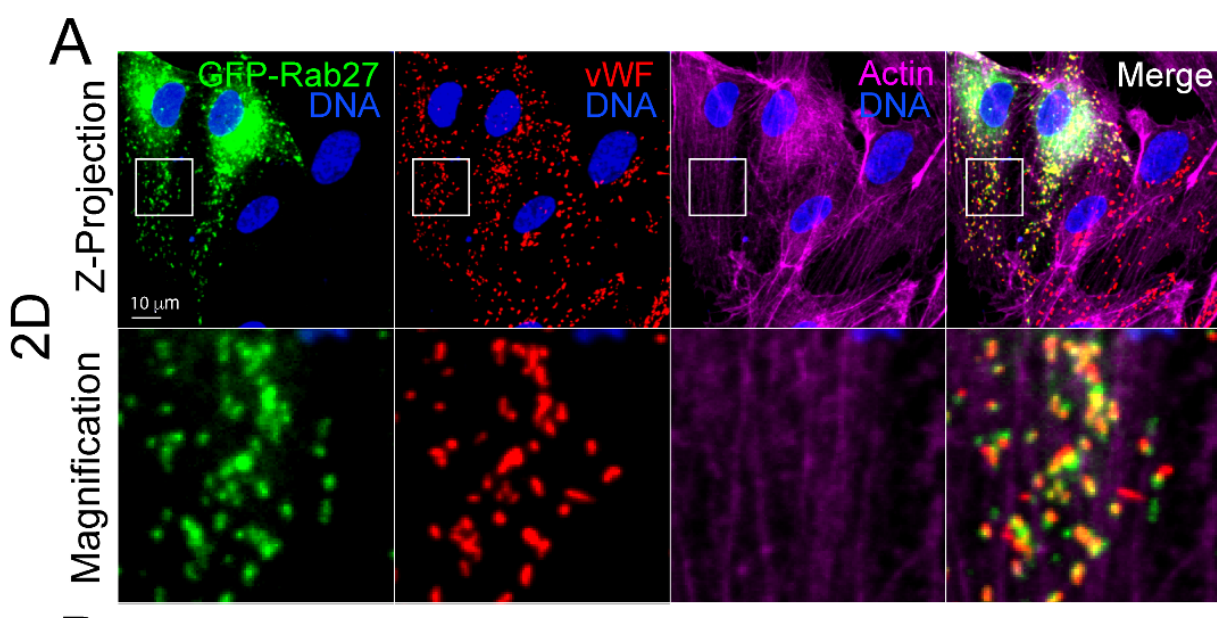

\section{C}
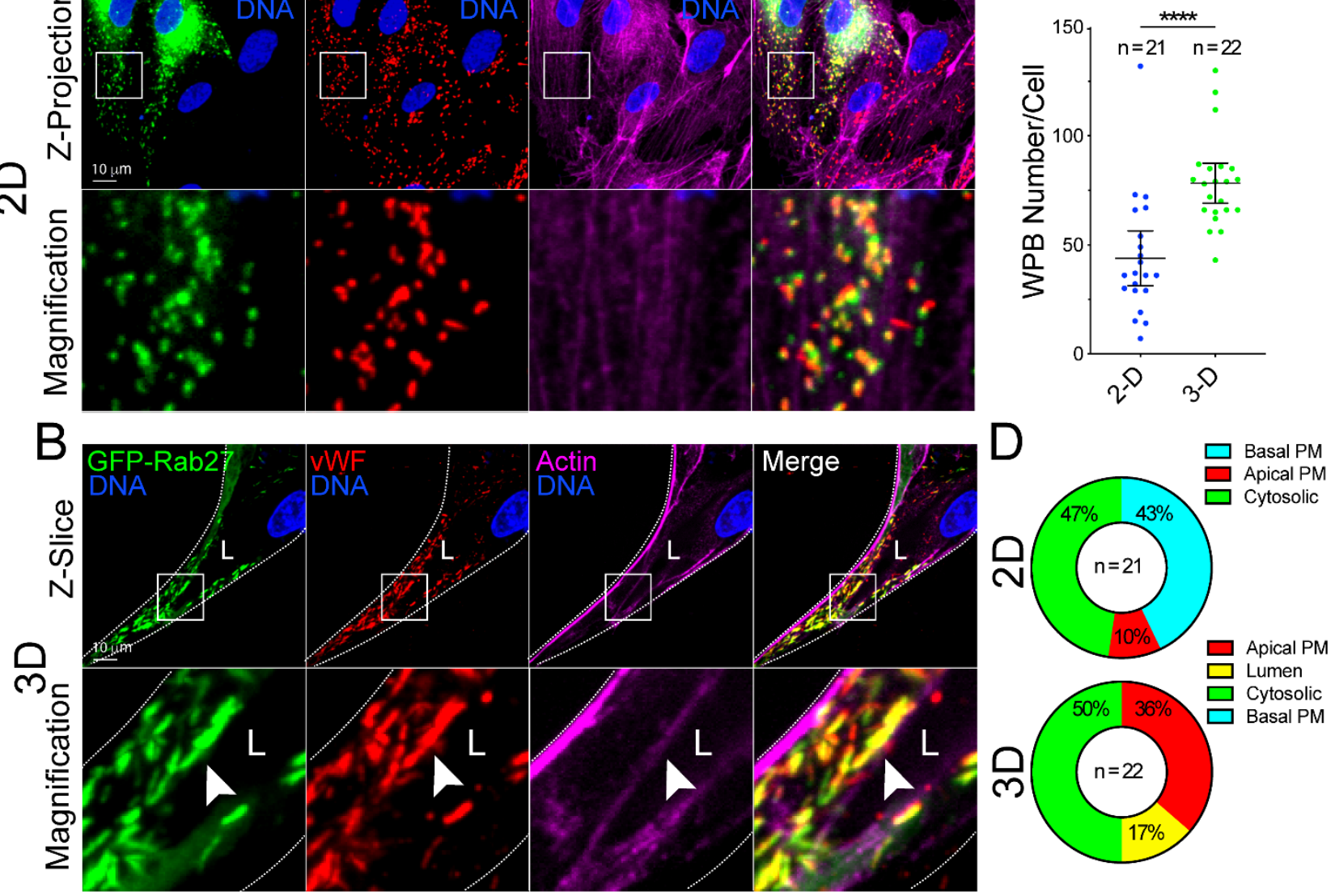

Figure 6. Imaging vWF exocytosis in 2D culture and 3D sprouts. (A) Representative images of endothelial cells in 2D culture expressing GFP-Rab27a and stained for von Willebrand Factor (VWF) and actin. (B) Representative images of a sprout expressing GFP-Rab27a and stained for VWF) and actin. White arrowhead denotes Weibel Palade Body accumulation. (C) Quantification of the number of vWF puncta between 2D culture and 3D sprouts. (D) Percentage of vWF accumulations ( $\geq 4$ vWF puncta) that localize to a particular cellular location. $P M=$ plasma membrane. $\mathrm{N}=$ number of cells. White boxes are areas of magnification and white dotted lines indicated sprout boundaries. L denotes lumen. Values are means +/- SEM; significance: ${ }^{* * * *} P<0.0001$. Statistical significance was assessed with an unpaired Students t-test. 

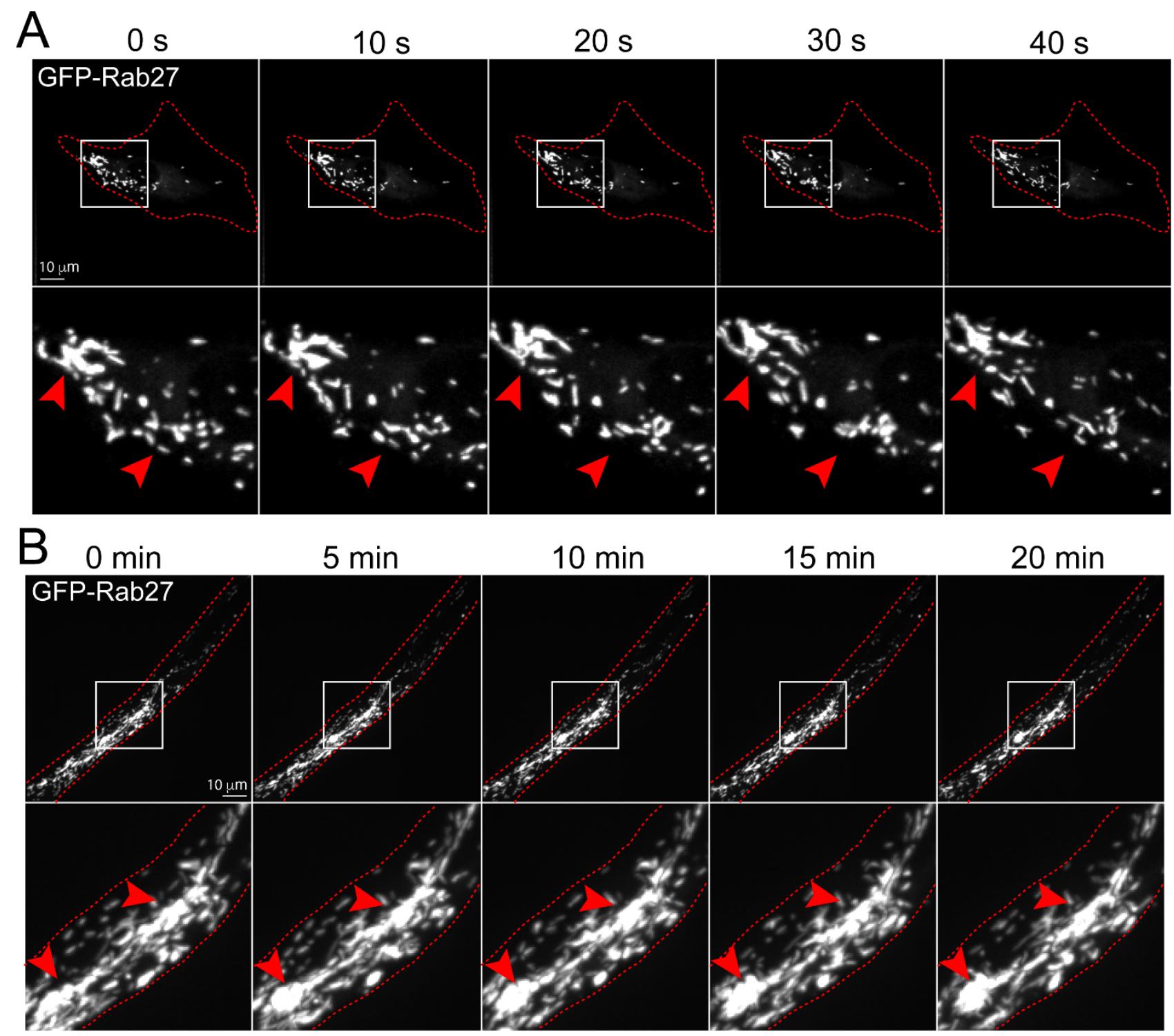

Figure 7. Live-imaging of Weibel Palade Body trafficking in 2D culture and 3D sprouting. (A) Live-imaging of endothelial cells expressing GFP-Rab27a (Weibel Palade Body marker) over time in 2D culture. Red arrowheads denote puncta accumulated at the leading edge of the cell. (B) Live-imaging of fibrin-bead generated sprout expressing GFP-Rab27a over time. Red arrowheads indicate accumulations of Weibel Palade Bodies at cell-cell interface. White boxes are areas of magnification and red botted lines indicated cell boundaries. $L$ denotes lumen. 


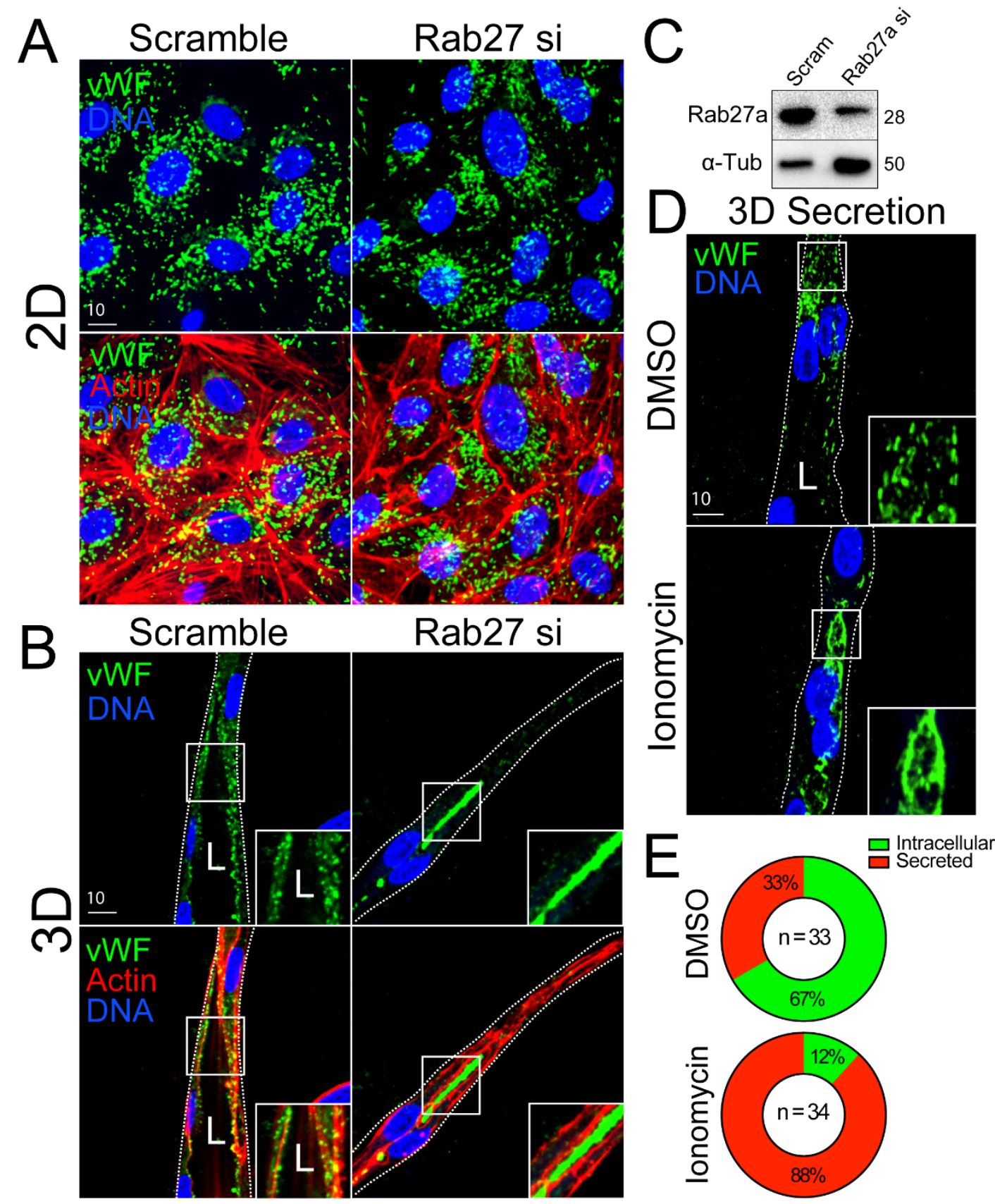

Figure 8. Monitoring exocytic events between 2D culture and 3D sprouting. (A) Representative 2D culture of endothelial cells stained for von Willebrand Factor (VWF) and actin treated with scramble or Rab27a-targeting siRNA (si). (B) Representative 3D fibrin-bead generated sprout stained for VWF and actin treated with scramble or Rab27a-targeting siRNA. (C) Western blot confirmation of Rab27a knockdown efficiency. (D) Representative 3D fibrin-bead generated sprout stained for VWF and treated with DMSO (vehicle) or ionomycin to induced Weibel Palade Body exocytosis. (E) Percentage of intracellular or lumen trapped vWF between indicated conditions. $\mathrm{N}=$ number of cells. White boxes are areas of magnification and white dotted lines indicated sprout boundaries. $L$ denotes lumen. 
752 Supplemental Materials: Capturing Membrane Trafficking Events During Angiogenic

753 Development in Vitro

754

755

756

757

758

Caitlin R. Francis ${ }^{1}$ and Erich J. Kushner ${ }^{1 *}$

${ }^{1}$ Department of Biological Sciences, University of Denver, Denver, CO; *Author for correspondence

\section{MAJOR RESOURCE TABLE}

\begin{tabular}{|c|c|c|}
\hline Reagent & Vendor & Catalog \# \\
\hline Poly-D-lysine & ThermoFisher & A3890401 \\
\hline $\begin{array}{l}\text { Polyethyleneamine Branched } \\
\text { (PEI) }\end{array}$ & Sigma-Aldrich & 408727 \\
\hline $\begin{array}{l}\text { Chloroquine Diphosphate } \\
\text { Crystalline (CQ) }\end{array}$ & Sigma-Aldrich & C6628-25G \\
\hline $\begin{array}{l}\text { Endothelial Cell Growth } \\
\text { Medium } 2\end{array}$ & PromoCell & C-22011 \\
\hline $\begin{array}{l}\text { DMEM, High Glucose, with L- } \\
\text { Glutamine }\end{array}$ & Genesee Scientific & $25-500$ \\
\hline $\begin{array}{l}\text { GenClone Fetal Bovine } \\
\text { Serum (FBS) }\end{array}$ & Genesee Scientific & $25-514$ \\
\hline $\begin{array}{l}\text { Penicillin-Streptomycin 100X } \\
\text { Solution }\end{array}$ & Genesee Scientific & P4333-100ML \\
\hline $\begin{array}{l}\text { DPBS, no Calcium, no } \\
\text { Magnesium }\end{array}$ & ThermoFisher & 14190250 \\
\hline $\begin{array}{l}\text { Trypsin-EDTA, o.25\% 1X, } \\
\text { phenol red }\end{array}$ & Genesee Scientific & $25-510$ \\
\hline $\begin{array}{l}\text { Paraformaldahyde } 20 \% \\
\text { Aqueous Sol. EM Grade }\end{array}$ & Electron Microscopy Sciences & 15713 \\
\hline Dimethyl Sulfoxide (DMSO) & Sigma-Aldrich & D2650-5X10ML \\
\hline $\begin{array}{l}\text { Silencer }{ }^{\mathrm{TM}} \text { Negative Control } \\
\text { No. } 1 \text { siRNA }\end{array}$ & ThermoFisher & AM4611 \\
\hline Rab27 siRNA & ThermoFisher & siRNA ID: s11695 \\
\hline Cytodex Microcarrier Beads & Sigma-Aldrich & C3275-10G \\
\hline $\begin{array}{l}\text { Fibrinogen Type 1-S from } \\
\text { Bovine Plasma }\end{array}$ & Sigma-Aldrich & F8630-1G \\
\hline
\end{tabular}




\begin{tabular}{|l|l|c|}
\hline $\begin{array}{l}\text { Thrombin from Bovine } \\
\text { Plasma }\end{array}$ & Sigma-Aldrich & T7513-500UN \\
\hline Aprotinin Protease Inhibitor & ThermoFisher & 78432 \\
\hline NHLF & Lonza & CC-2512 \\
\hline HEK 293-A & ThermoFisher & R70507 \\
\hline
\end{tabular}

\begin{tabular}{|c|c|c|c|}
\hline \multicolumn{4}{|l|}{ ANTIBODIES } \\
\hline Target Antigen & $\begin{array}{l}\text { Vendor or } \\
\text { Source }\end{array}$ & $\begin{array}{l}\text { Catalog No./ } \\
\text { Clone }\end{array}$ & $\begin{array}{l}\text { Working } \\
\text { Concentration }\end{array}$ \\
\hline Moesin & Abcam & ab52490 & $\begin{array}{l}0.05 \mathrm{ug} / \mathrm{mL} \\
(1: 1000)\end{array}$ \\
\hline VE-Cadherin & ThermoFischer & $14-1441-82$ & $\begin{array}{l}0.5 \mathrm{ug} / \mathrm{mL} \\
(1: 1000)\end{array}$ \\
\hline Podocalyxin & R\&D & AF1658 & $15 \mathrm{ug} / \mathrm{mL}(1: 200)$ \\
\hline $\begin{array}{l}\text { Von Willebrand } \\
\text { Factor }\end{array}$ & Abcam & ab6994 & $\begin{array}{l}10 \mathrm{ug} / \mathrm{mL} \\
(1: 1000)\end{array}$ \\
\hline Beta-1 Integrin & Abcam & Ab230291 & $\begin{array}{l}\text { 10ug/mL } \\
(1: 1000)\end{array}$ \\
\hline $\begin{array}{l}\text { Alexa Fluor }{ }^{\mathrm{TM}} \\
488 \text { Phalloidin }\end{array}$ & ThermoFisher & A12379 & 1 uM (1:200) \\
\hline $\begin{array}{l}\text { Alexa Fluor }{ }^{\mathrm{TM}} \\
647 \text { Phalloidin }\end{array}$ & ThermoFisher & A22287 & 1 uM (1:200) \\
\hline $\begin{array}{l}\text { Alexa Fluor }{ }^{\top \mathrm{M}} \\
555 \text { Phalloidin }\end{array}$ & ThermoFisher & A34055 & 1 uM (1:200) \\
\hline $\begin{array}{l}\text { Goat anti-Rabbit } \\
\text { IgG }(\mathrm{H}+\mathrm{L}) \\
\text { Secondary } \\
\text { Antibody, Alexa } \\
\text { Fluor } 488\end{array}$ & ThermoFisher & A11008 & $1 \mathrm{ug} / \mathrm{mL}(1: 500)$ \\
\hline $\begin{array}{l}\text { Donkey anti- } \\
\text { Rabbit IgG }(\mathrm{H}+\mathrm{L}) \\
\text { Secondary } \\
\text { Antibody, Alexa } \\
\text { Fluor } 555\end{array}$ & ThermoFisher & A31572 & $1 \mathrm{ug} / \mathrm{mL}(1: 500)$ \\
\hline
\end{tabular}




\begin{tabular}{|l|l|l|l|}
\hline $\begin{array}{l}\text { Donkey anti-goat } \\
\text { IgG }(\mathrm{H}+\mathrm{L}) \\
\text { Secondary } \\
\text { Antibody, Alexa } \\
\text { Flour 488 }\end{array}$ & ThermoFisher & A11055 & $1 \mathrm{ug} / \mathrm{mL}(1: 500)$ \\
\hline $\begin{array}{l}\text { Donkey anti- } \\
\text { Goat lgG (H+L) } \\
\text { Cross-Adsorbed } \\
\text { Secondary } \\
\text { Antibody, Alexa } \\
\text { Fluor 555 }\end{array}$ & ThermoFisher & A21432 & \\
\hline $\begin{array}{l}\text { Chicken anti- } \\
\text { Rabbit IgG (H+L) } \\
\text { Cross-Adsorbed } \\
\text { Secondary } \\
\text { Antibody, Alexa } \\
\text { Fluor 647 }\end{array}$ & ThermoFisher & A21443 & \\
\hline
\end{tabular}

\section{SUPPLEMENTAL MOVIES}

Movie 1. Live-imaging of pHluorin-podocalyxin in 2D culture. Intervals are $1 \mathrm{frame} /$ second for 2 minutes.

775

776

777

778

779

780

781

782

783

784

785

786

787

788

789

790

791

792

Movie 2. Live-imaging of pHluorin-podocalyxin in 3D sprouts. Intervals are 1 frame/ 2 minutes for 20 minutes.

Movie 3. Live-imaging of GFP-Rab35 in 2D culture. Intervals are 1 frame/ second for 2 minutes.

Movie 4. Live-imaging of GFP-Rab35 in 3D sprouts. Intervals are 1 frame/ 2 minutes for 20 minutes.

Movie 5. Live-imaging of GFP-Rab27a in 2D culture. Intervals are 1 frame/ minute for 10 minutes.

Movie 6. Live-imaging of GFP-Rab27a in 2D sprouts. Intervals are 1 frame/2minutes for 20 minutes. 